\title{
Effect of anticoagulants on the risk of delayed bleeding after colorectal endoscopic submucosal dissection
}

\section{(ㄷ)(1) $\Theta$}

\section{Authors}

Hideharu Ogiyama ${ }^{1}$, Takuya Inoue ${ }^{2}$, Akira Maekawa ${ }^{3}$, Shunsuke Yoshii ${ }^{4}$, Shinjiro Yamaguchi ${ }^{5}$, Kengo Nagai ${ }^{6}$, Masashi Yamamoto $^{7}$, Satoshi Egawa ${ }^{8}$, Masayoshi Horimoto ${ }^{9}$, Hiroyuki Ogawa ${ }^{10}$, Akihiro Nishihara ${ }^{11}$, Masato Komori ${ }^{12}$, Takashi Kizu $^{13}$, Shusaku Tsutsui ${ }^{1}$, Yoshiki Tsujii ${ }^{4}$, Yoshito Hayashi ${ }^{4}$, Hideki lijima ${ }^{4}$, Tetsuo Takehara ${ }^{4}$

Institutions

1 Departments of Gastroenterology and Hepatology, Itami City Hospital, Itami, Japan

2 Department of Gastroenterology and Hepatology, Osaka General Medical Center, Osaka, Japan

3 Department of Gastrointestinal Oncology, Osaka International Cancer Institute, Osaka, Japan

4 Department of Gastroenterology and Hepatology, Osaka University Graduate School of Medicine, Suita, Japan

5 Department of Gastroenterology, Kansai Rosai Hospital, Amagasaki, Japan

6 Department of Gastroenterology and Hepatology, Suita Municipal Hospital, Suita, Japan

7 Department of Gastroenterology, Toyonaka Municipal Hospital, Toyonaka, Japan

8 Department of Gastroenterology, Osaka Police Hospital, Osaka, Japan

9 Department of Gastroenterology, Saiseikai Senri Hospital, Suita, Japan

10 Department of Gastroenterology, Nishinomiya Municipal Central Hospital, Nishinomiya, Japan

11 Department of Gastroenterology, Minoh City Hospital, Minoh, Japan

12 Department of Gastroenterology, Hyogo Prefectural Nishinomiya Hospital, Nishinomiya, Japan

13 Department of Gastroenterology, Yao Municipal Hospital, Yao, Japan

submitted 22.5.2020

accepted after revision $\quad 30.7 .2020$

\section{Bibliography}

Endoscopy International Open 2020; 08: E1654-E1663

DOI 10.1055/a-1244-2097

ISSN 2364-3722

(C) 2020. The Author(s).

This is an open access article published by Thieme under the terms of the Creative Commons Attribution-NonDerivative-NonCommercial License, permitting copying and reproduction so long as the original work is given appropriate credit. Contents may not be used for commecial purposes, or adapted, remixed, transformed or built upon. (https://creativecommons.org/licenses/by-nc-nd/4.0/)
Corresponding author

Tetsuo Takehara, MD, PhD, Department of Gastroenterology and Hepatology, Osaka University Graduate School of Medicine, 2-2 Yamadaoka Suita, Osaka 565-0871, Japan

Fax: +81-6-6879-3629

takehara@gh.med.osaka-u.ac.jp

\section{ABSTRACT}

Background and study aims In patients receiving antithrombotic therapy, the risks of delayed bleeding after endoscopic procedures for gastrointestinal neoplasms become a major problem. Few reports have shown the effects of delayed bleeding in patients taking anticoagulants after colorectal endoscopic submucosal dissection (ESD). This study aimed to evaluate the delayed bleeding events after colorectal ESD in patients receiving anticoagulant therapy.

Patients and methods We retrospectively analyzed 87 patients taking anticoagulants who underwent colorectal ESD from April 2012 to December 2017 at 13 Japanese institutions participating in the Osaka Gut Forum. Among these patients, warfarin users were managed with heparin bridge therapy (HBT), continued use of warfarin, a temporary switch to direct oral anticoagulation (DOAC), or withdrawal of warfarin, and DOAC users were managed with DOAC discontinuation with or without HBT. We investigated the occurrence rate of delayed bleeding and compared the rates between warfarin and DOAC users.

Results The delayed bleeding rate was $17.2 \%$ among all patients. The delayed bleeding rate was higher in DOAC users than in warfarin users $(23.3 \%$ vs. $11.4 \%, P=0.14)$, although no statistically significant difference was observed. In DOAC users, the delayed bleeding rates for dabigatran, rivaroxaban, apixaban, and edoxaban users appeared similar (30\%, $18.2 \%, 22.2 \%$, and $25 \%$, respectively). The onset of delayed bleeding in both warfarin and DOAC users was late, averaging 6.9 and 9.4 days, respectively.

Conclusions Among patients taking anticoagulants, the risk of delayed bleeding after colorectal ESD was relatively high and the onset of delayed bleeding was late. 


\section{Introduction}

Colorectal endoscopic submucosal dissection (ESD) has a high en bloc resection rate and is minimally invasive. It is widely performed for large superficial colorectal tumors. One of the major complications of colorectal ESD is delayed bleeding. The rate of delayed bleeding ranges from $1 \%$ to $5 \%$ in previous reports with $>100$ cases [1-4].

Given that the elderly population has increased, the number of patients receiving antithrombotic therapy with anticoagulants has also increased worldwide. Thromboembolic events such as cerebrovascular events that occur during the withdrawal period from anticoagulants can be life-threatening. A previous report demonstrated that patients taking a vitamin $\mathrm{K}$ antagonist such as warfarin have approximately a $1 \%$ stroke risk per endoscopic procedure after the discontinuation of the drug [5]. Therefore, it is recommended that the duration of withholding antithrombotic drugs should be minimized during endoscopic procedures. Several risk factors for delayed bleeding after colorectal ESD have been reported and include tumor location in the rectum [3,6-9], three or more arterial bleeding events during an ESD procedure [7], exposure to low-dose aspirin (LDA) even if LDA was discontinued [10], and exposure to antiplatelet agents, except for aspirin alone [9].

Many reports have demonstrated that colonoscopic polypectomy in patients receiving anticoagulation therapy has a high risk of delayed bleeding [11-13]. Conversely, in colorectal ESD, few reports have demonstrated a high incidence of delayed bleeding in patients taking anticoagulants, but these studies were conducted at a single institution and involved a small number of cases $[8,14,15]$. This multicenter study aimed to evaluate the rate of delayed bleeding in patients taking anticoagulants after colorectal ESD for colorectal neoplasms.

\section{Patients and methods}

\section{Patients and study design}

This retrospective multicenter cohort study was performed at one academic and 12 tertiary hospitals participating in the Osaka Gut Forum (OGF1913). We enrolled patients on anticoagulant therapy who underwent colorectal ESD for colorectal neoplasms, except for neuroendocrine tumor, from April 2012 to December 2017. Delayed bleeding was defined as clinical evidence of bleeding, such as massive hematochezia or a decrease in hemoglobin level $>2 \mathrm{~g} / \mathrm{dL}$, within 30 days after ESD.

\section{Inclusion criteria}

indications for colorectal ESD were determined by endoscopists in each hospital based on Japan Gastroenterological Endoscopy Society (JGES) guidelines [16]. We included patients taking anticoagulants, including warfarin potassium or direct oral anticoagulants (DOACs) (dabigatran, rivaroxaban, apixaban, and edoxaban).

\section{Exclusion criteria}

We excluded patients whose post-ESD ulcers achieved prophylactic clip closure, which might have affected delayed bleeding. The patients who underwent an emergent surgery for perforations were also excluded.

\section{Management of anticoagulants and antiplatelet agents}

Timing of discontinuation and restart of oral anticoagulants and antiplatelet agents were mostly according to JGES guidelines $[17,18]$. In summary, warfarin users were managed with heparin bridge therapy (HBT), continued warfarin therapy, a temporary switch to DOAC (DOAC replacement), or warfarin withdrawal without HBT. These methods, other than warfarin withdrawal without HBT, follow the JGES guidelines $[17,18]$. Regarding HBT for warfarin users, warfarin was suspended for 3 to 5 days before ESD, and then unfractionated heparin was initiated intravenously and the dose was adjusted to attain the required activated partial thromboplastin time [17]. Heparin sodium was discontinued for at least 3 hours before the ESD procedure. On the day after ESD, heparin sodium and warfarin were restarted after confirming absence of hematochezia or development of anemia. When the international normalized ratio of prothrombin time (PT-INR) had reached approximately 1.50 , heparin sodium was discontinued. In continued warfarin therapy, ESD was performed when the PT-INR was maintained within the therapeutic range on the day before ESD or the day of ESD.

DOAC users were managed with or without HBT. DOAC with HBT was suspended for 24 to 48 hours before ESD; HBT was introduced later, and once hemostasis was confirmed after ESD, DOAC was resumed [17]. The newer revised JGES guideline recommend that DOAC be managed by withdrawal on the day of treatment [18]. The discontinuation period for DOAC varies slightly, according to the JGES, the American Society for Gastrointestinal Endoscopy (ASGE) [19], the British Society of Gastroenterology, and the European Society of Gastrointestinal Endoscopy guidelines [20]. This period also depends on each DOAC and renal function. In this study, we regarded DOAC users with $>3$ days of discontinuation as patients who did not follow guidelines. Therefore, we also divided the DOAC users without HBT into the following two groups: patients with 0 to 3 days of discontinuation (short-term discontinuation) and those with $>3$ days of discontinuation (long-term discontinuation). Management of anticoagulants and antiplatelet agents was determined after consultation with experts, such as cardiologists.

\section{Data collection}

We collected data on the following variables: patient characteristics before ESD (age, sex, name of anticoagulants and antiplatelet drugs used, and reasons for anticoagulant use), and laboratory data (platelet, PT-INR, creatinine, and estimated glomerular filtration rate [eGFR]). We also collected information regarding anticoagulant withdrawal and details of endoscopic procedures, delayed bleeding, and length of hospitalization 
- Table 1 Clinical characteristics of all patients and in patients with non-delayed and delayed bleeding.

\begin{tabular}{|c|c|c|c|c|}
\hline & Total $\mathbf{n}=\mathbf{8 7}$ & $\begin{array}{l}\text { Non-delayed bleeding } \\
(\mathrm{n}=72)\end{array}$ & $\begin{array}{l}\text { Delayed bleeding } \\
(n=15)\end{array}$ & $P$ value \\
\hline Age (years, mean $\pm S D$ ) & $74.5 \pm 7.6$ & $74.9 \pm 7.6$ & $72.5 \pm 7.7$ & 0.28 \\
\hline Sex (male/female) & $65 / 22$ & $52 / 20$ & $13 / 2$ & 0.24 \\
\hline Platelet $\left(\times 10^{4}\right.$, mean \pm SD $)$ & $20.4 \pm 5.7$ & $20.7 \pm 5.5$ & $18.8 \pm 6.4$ & 0.23 \\
\hline eGFR $\left(\mathrm{mL} / \mathrm{min} / 1.73 \mathrm{~m}^{2}\right.$, mean $\left.\pm \mathrm{SD}\right)$ & $58.5 \pm 21.8$ & $57.5 \pm 22.2$ & $63.4 \pm 19.7$ & 0.34 \\
\hline PT-INR (mean $\pm S D$ ) & $1.52 \pm 0.53$ & $1.52 \pm 0.51$ & $1.53 \pm 0.62$ & 0.94 \\
\hline Anticoagulants (warfarin/DOAC) & $44 / 43$ & $39 / 33$ & $5 / 10$ & 0.14 \\
\hline Antiplatelet drugs, n (\%) & $21(24.1)$ & $15(20.8)$ & $6(40.0)$ & 0.11 \\
\hline Heparin bridge therapy, n (\%) & $49(56.3)$ & $40(55.6)$ & $9(60.0)$ & 0.75 \\
\hline Tumor location (colon/rectum) & $65 / 22$ & $59 / 13$ & $6 / 9$ & 0.001 \\
\hline Macroscopic features (LST-G/LST-NG/protruded) & $35 / 36 / 16$ & $27 / 32 / 13$ & $8 / 4 / 3$ & 0.42 \\
\hline
\end{tabular}

and fasting. eGFR was calculated using the serum creatinine lev$\mathrm{el}$, age, and sex, according to the following Japanese formula: eGFR $\left(\mathrm{mL} / \mathrm{min} / 1.73 \mathrm{~m}^{2}\right)=194 \times \mathrm{Cr}-1.094 \times$ age $-0.287(\times 0.739$ if female) [21].

\section{Measured outcomes}

We analyzed characteristics and clinical outcomes of all patients and compared the data between the warfarin and DOAC groups. We investigated the delayed bleeding rate in all patients and compared the rate between the two groups and among the management strategies of the anticoagulants. We also analyzed the association between delayed bleeding and the patient characteristics and other clinical outcomes.

\section{Ethics}

This study was performed in accordance with the Declaration of Helsinki and Ethical Guidelines for Medical and Health Research Involving Human Subjects of the Ministry of Health, Labor and Welfare, and the Ministry of Education, Cultural, Sports, Science, and Technology in Japan. This study was approved by the ethics committees of Osaka University Hospital and all the participating hospitals. All patients were provided opportunities to decline participation in this study by the opt-out method of each hospital. Moreover, the requirement for obtaining informed consent was waived.

\section{Statistical analysis}

Continuous and categorical variables are presented as means \pm standard deviation, medians and interquartile range (IQR), and proportions, respectively. Data were compared using the Student's $t$-test, Wilcoxon rank-sum test, chi-square test, or Fisher's exact test, as appropriate. $P<0.05$ was considered statistically significant. Statistical analysis was performed using JMP software (ver. 12.2.0; SAS Institute Inc., Cary, North Carolina, United States).

\section{Results}

We enrolled 107 patients from 13 Japanese institutions belonging to the Osaka Gut Forum from April 2012 to December 2017. Patients who had prophylactic clip closure of lesions $(n=$ $18)$ and those requiring operation due to perforations $(n=2)$ were excluded from the analysis ( $\triangleright$ Supplementary Fig. 1 ).

- Table 1 shows the clinical characteristics of the whole cohort and patients with non-delayed and delayed bleeding. The proportion of patients with tumors located in the rectum was significantly higher in the delayed bleeding group than in the non-delayed bleeding group $(P=0.001)$. We found no significant difference in the delayed bleeding rate between the warfarin and DOAC groups or between patients with and without HBT. Antiplatelet agents were used by $24.1 \%$ of patients. The delayed bleeding rate in all cases was $17.2 \%$ ( $\triangleright$ Table 2 ). This rate was $17.1 \%(13 / 76)$ when we excluded the patients with the different management techniques for warfarin discontinuation without HBT in the warfarin group and long-term discontinuation in the DOAC group, who were not following any guidelines. The proportion of patients with delayed bleeding requiring endoscopic hemostasis was $12.6 \%$. Transfusion was required in three patients $(3.4 \%)$. Both hospitalization and fasting duration were longer in patients with delayed than those with non-delayed bleeding (median [IQR], 15 [10-25] vs. 10 [8-14] days, 4 [3-7] vs. 2 [2-2] days, respectively).

- Table 3 shows the clinical characteristics of the warfarin and DOAC groups. Among the 87 patients, the number taking warfarin and DOAC was 44 and 43 , respectively. The rate of laterally spreading tumors of non-granular type was higher in the warfarin group than in the DOAC group $(P=0.01)$. The most common reason for anticoagulant use was atrial fibrillation in both the warfarin and DOAC groups. Clinical outcomes are shown in $>$ Table 4 . The delayed bleeding rate was higher in the DOAC group than in the warfarin group ( $23.3 \%$ vs. $11.4 \%)$, 
- Table 2 Clinical outcomes of all patients and in patients with non-delayed and delayed bleeding.

\begin{tabular}{|c|c|c|c|c|}
\hline & $\begin{array}{l}\text { Total } \\
(n=87)\end{array}$ & $\begin{array}{l}\text { Non-delayed bleeding } \\
(\mathrm{n}=72)\end{array}$ & $\begin{array}{l}\text { Delayed bleeding } \\
(n=15)\end{array}$ & $P$ value \\
\hline Resected specimen size $(\mathrm{mm}$, mean $\pm \mathrm{SD})$ & $35.4 \pm 13.2$ & $34.9 \pm 12.5$ & $37.9 \pm 16.4$ & 0.42 \\
\hline Resected tumor size $(\mathrm{mm}$, mean $\pm \mathrm{SD}$ ) & $27.6 \pm 11.2$ & $26.7 \pm 10.0$ & $31.7 \pm 15.2$ & 0.12 \\
\hline $\begin{array}{l}\text { Histology (adenoma/mucosal cancer/submucosal } \\
\text { cancer), } \mathrm{n}(\%)\end{array}$ & $38 / 36 / 11$ & $36 / 27 / 9$ & $3 / 10 / 2$ & 0.08 \\
\hline Procedure time (min, mean $\pm S D$ ) & $106.5 \pm 72.9$ & $102.6 \pm 65.0$ & $125.2 \pm 103.1$ & 0.28 \\
\hline Delayed bleeding, n (\%) & $15(17.2)$ & & & \\
\hline Delayed bleeding with endoscopic hemostasis, $\mathrm{n}(\%)$ & $11(12.6)$ & - & $11(73.3)$ & - \\
\hline Transfusion, $\mathrm{n}(\%)$ & $3(3.4)$ & - & $3(20.0)$ & - \\
\hline Thromboembolism, n (\%) & $0(0)$ & $0(0)$ & $0(0)$ & 1.0 \\
\hline Length of hospitalization, median (IQR), days & $11(8-15)$ & $10(8-14)$ & $15(10-25)$ & 0.001 \\
\hline Fasting duration, median (IQR), days & $2(2-3)$ & $2(2-2)$ & $4(3-7)$ & 0.0002 \\
\hline
\end{tabular}

- Table 3 Clinical characteristics of the warfarin and DOAC groups.



although no statistically significant difference was observed. The result was almost the same $(21.6 \%$ [8/37] vs. $12.8 \%$ [5/ 39]) when we excluded patients who had not followed the guidelines.
- Fig. 1 a shows delayed bleeding rates in the warfarin group for the four management strategies according to discontinuation and replacement of warfarin and of the DOAC group with three strategies. In the warfarin group, delayed bleeding rates in patients with discontinued warfarin without HBT, discontin- 


\begin{tabular}{|c|c|c|c|}
\hline & $\begin{array}{l}\text { Warfarin } \\
n=44\end{array}$ & $\begin{array}{l}\text { DOAC } \\
n=43\end{array}$ & $P$ value \\
\hline Resected specimen size $(\mathrm{mm}$, mean $\pm \mathrm{SD})$ & $38.0 \pm 13.0$ & $32.8 \pm 13.0$ & 0.07 \\
\hline Resected tumor size (mm, mean \pm SD) & $29.0 \pm 10.3$ & $26.1 \pm 11.9$ & 0.23 \\
\hline Histology (adenoma/mucosal cancer/submucosal cancer) & $19 / 21 / 4$ & $20 / 16 / 7$ & 0.47 \\
\hline Procedure time (min, mean \pm SD) & $100.2 \pm 60.1$ & $112.9 \pm 84.0$ & 0.42 \\
\hline Delayed bleeding, n (\%) & $5(11.4)$ & $10(23.3)$ & 0.14 \\
\hline Delayed bleeding with endoscopic hemostasis, n (\%) & $3(6.8)$ & $8(18.6)$ & 0.12 \\
\hline Transfusion, n (\%) & $0(0)$ & $3(7.0)$ & 0.12 \\
\hline Thromboembolism, n (\%) & $0(0)$ & $0(0)$ & 1.0 \\
\hline Length of hospitalization, median (IQR), days & $14.0(10.0-16.0)$ & $8.5(6.3-12.8)$ & 0.39 \\
\hline Fasting duration, median (IQR), days & $2.0(2.0-3.0)$ & $2.0(2.0-3.8)$ & 0.75 \\
\hline
\end{tabular}

ued warfarin with HBT, continued warfarin therapy, and DOAC replacement were $0 \%, 12.1 \%, 33.3 \%$, and $0 \%$, respectively. In the DOAC group, the delayed bleeding rates in patients with $\mathrm{HBT}$, with short-term discontinuation without $\mathrm{HBT}$, and with long-term discontinuation without HBT were $33.3 \%, 13.6 \%$, and $33.3 \%$, respectively. There was no significant difference across the different management strategies.

- Fig. 1b shows the delayed bleeding rate in each DOAC type. The delayed bleeding rates for dabigatran, rivaroxaban, apixaban, and edoxaban were $30 \%, 18.2 \%, 22.2 \%$, and $25 \%$, respectively. There was no significant difference in the bleeding rate across the types of DOACs.

The 15 delayed bleeding cases are shown in $>$ Table 5 . Delayed bleeding occurred more than once in three patients. Moreover, delayed bleeding occurred four times in one patient and twice in two patients.

- Fig. 2 shows the onset of delayed bleeding after colorectal ESD, including every delayed bleeding event in patients who experienced several events. Median days from ESD to delayed bleeding in the warfarin and DOAC groups were 10 (range: 219 ) and 4 (range: 1-22) days, respectively. There was no significant difference between the two groups.

\section{Discussion}

In this study, the delayed bleeding rate after colorectal ESD in patients taking anticoagulants was relatively high at $17.2 \%$ compared to the previously reported rates (1\%-5\%) [1-4]. A report from Odagiri et al. was based on national impatient data from the Japanese Diagnosis Procedure Combination database, containing data of our institutions [4]. The composition of the institutions associated with those data resembles that of our institutions, which consist of one academic and 12 tertiary hospitals. Therefore, the delayed bleeding rates among all patients in our institutions, which we could not evaluate in this study, is estimated to be similar to the $4.4 \%$ rate in Odagiri's re- port. In addition, the proportion of patients requiring blood transfusion due to severe bleeding was high at 3.4\%. Our data showing the high occurrence rate of delayed bleeding were similar to the rates reported in previous single-center, retrospective studies that included a small number of patients taking anticoagulants $[8,14,15,22]$. Our study is the first multicenter investigation to present detailed data on a high delayed bleeding rate in patients receiving anticoagulant therapy who underwent colorectal ESD. Although previous studies did not consider whether prophylactic clip closure was performed, we excluded lesions for which it was used to ensure accurate analysis of post-ESD bleeding.

In this study, the delayed bleeding rate was higher in the DOAC group than in the warfarin group, although the difference was not statistically significant. That may be due to the pharmacological effects of DOACs. DOACs may not be completely absorbed in the gastrointestinal tract, and the non-absorbed active anticoagulant is believed to promote gastrointestinal bleeding [23]. Three previous reports have shown delayed bleeding rates for colorectal ESD in patients taking DOAC. Harada et al. reported that the rate of delayed bleeding was higher in the DOAC group than in the warfarin group, with the difference not reaching statistical significance (16\% [4/25] vs. $7.7 \%$ [6/26]) [22]. Yamashita et al. reported that the rate of delayed bleeding in patients taking DOAC was $22.2 \%$ (2/9) [15]. In these reports, the number of patients taking DOAC was small. Nagata et al. reported that the delayed bleeding rates for colorectal ESD were no different between the warfarin and DOAC groups ( $15.3 \%$ vs. $13.2 \%$ ) recruited from the Japanese Diagnosis Procedure Combination database [24]. However, that report did not include detailed information regarding the drugs, such as drug cessation, or the lesions. Therefore, there is no consensus regarding the risk of bleeding associated with colorectal ESD in DOAC and warfarin users. A previous meta-analysis showed that DOAC users have a higher risk of gastrointestinal bleeding than warfarin users, but this was unrelated to the endoscopic 


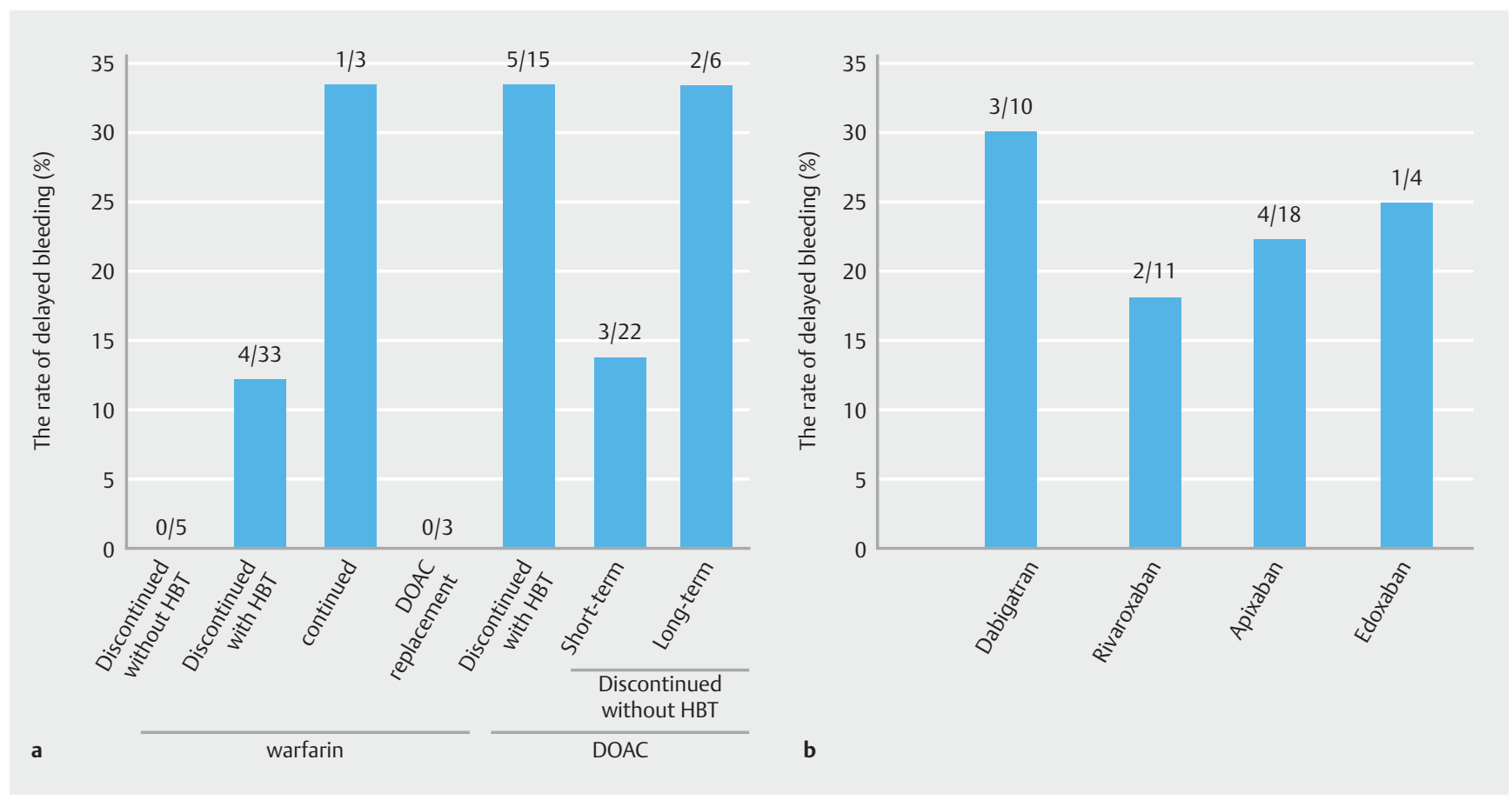

Fig. 1 Rates of delayed bleeding according to the management strategies and DOAC types. a Rates of delayed bleeding in the warfarin group with four management strategies and in the DOAC group with three strategies. In the warfarin group, the rate of delayed bleeding in patients with discontinuation of treatment without HBT, discontinuation of treatment with HBT, continued warfarin therapy, and DOAC replacement was $0 \%, 12.1 \%, 33.3 \%$, and $0 \%$, respectively. In the DOAC group, the rate of delayed bleeding in patients with HBT, with short-term ( $0-3$ days) discontinuation of treatment without HBT and with long-term ( $>3$ days) discontinuation of treatment without HBT was $33.3 \%$, $13.6 \%$ and $33.3 \%$, respectively. b Rate of delayed bleeding in each DOAC type. Delayed bleeding rates in the dabigatran, rivaroxaban, apixaban, and edoxaban groups were $30 \%, 18.2 \%, 22.2 \%$, and $25 \%$, respectively. DOAC, direct oral anticoagulant; HBT, heparin bridge therapy.

procedures [25]. Further studies are needed to clarify whether there is a difference in the delayed bleeding risk between warfarin and DOAC users.

The average onset from colorectal ESD to delayed bleeding was 9.4 and 6.9 days in the DOAC and warfarin groups, respectively. The time to delayed bleeding was relatively long when compared to that after endoscopic resection without anticoagulant drugs, which mainly occurs within 1 week [16]. Yoshio et al. reported that delayed bleeding in gastric ESD occurred later in patients with HBT than in those without HBT ( $8.0 \pm 5.7$ vs. $3.8 \pm 4.1$ days) [26]. The rate of late-onset delayed bleeding may be high in patients taking DOAC and those receiving HBT; late-onset delayed bleeding may lead to longer hospitalization.

Several reports have shown that HBT in warfarin users is a risk factor for delayed bleeding after colonoscopic polypectomy [27-29] and after gastric ESD [26, 30]. In this study, the delayed bleeding rate was relatively low in the warfarin group with HBT $(12.1 \%)$ compared with the DOAC group (23.2\%). Yamashita et al. reported that the delayed bleeding rate in patients taking warfarin with HBT was $26.3 \%$ (5/19) [15], whereas Yoshida et al. reported that the rate was $0 \%(0 / 14)$ [14]. Therefore, it is unclear whether HBT in warfarin users is a risk factor for delayed bleeding. In the DOAC group, patients with HBT had a higher rate of delayed bleeding than those without HBT (not statistically significant). Nagata et al. also reported that DOAC users with HBT who underwent therapeutic endoscopy had a higher delayed bleeding rate than those without HBT [24]. The complications of HBT, such as delayed bleeding and thromboembolism, in DOAC patients were reported in previous reports [31,32]. DOAC with HBT in colorectal ESD might be limited to patients who cannot restart DOAC within 24 hours, which is recommended in the ASGE guideline [19].

The JGES guideline, published in 2018, showed that continuation of warfarin during endoscopic resection is an option when the PT-INR is confirmed to be within the therapeutic range [18]. Harada et al. reported that delayed bleeding after colorectal ESD in patients with continued warfarin occurred in two of 14 patients [22]. In our study, delayed bleeding occurred in one of three patients. These results show that colorectal ESD with continued warfarin therapy also may have a high risk for delayed bleeding.

In this study, none of the patients had thromboembolism. Several reports have shown that the rate of thromboembolic events in the perioperative period in patients taking anticoagulants ranges from $0.2 \%$ to $0.4 \%$ [33-35]. A short withdrawal period for anticoagulants under the JGES guideline may be feasible to minimize thromboembolic events. In this study, delayed bleeding in the DOAC group with long-term discontinuation that did not follow the guidelines occurred in two of six patients. Radaelli et al. reported that delayed resumption did not result in a decreased bleeding risk in gastrointestinal endoscopy [33], which was consistent with the results of the present 


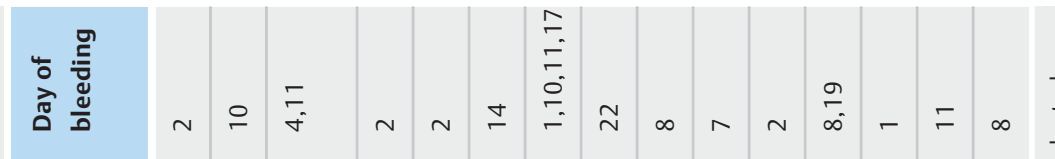

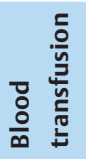
$\stackrel{y}{\rightleftharpoons}$

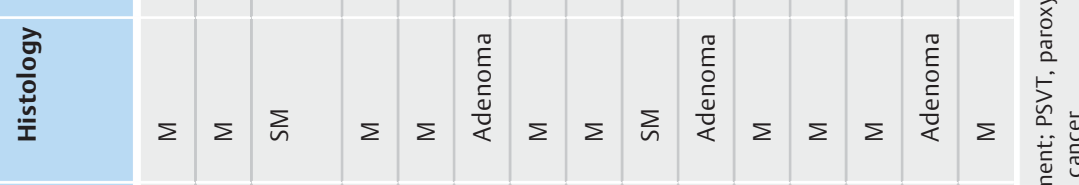

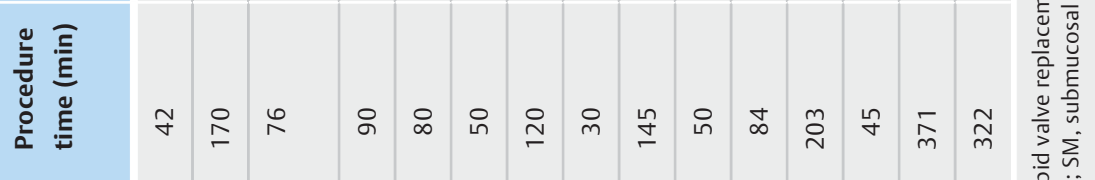

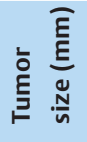

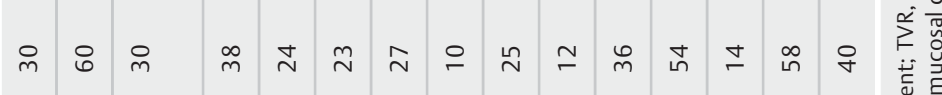

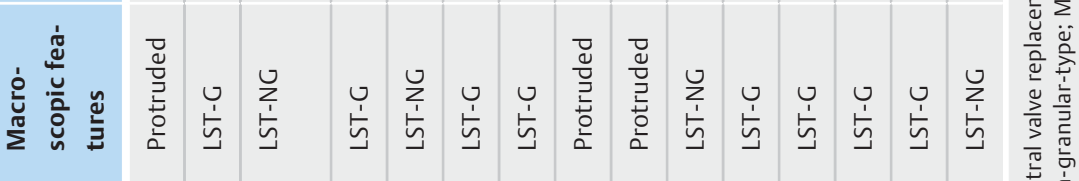

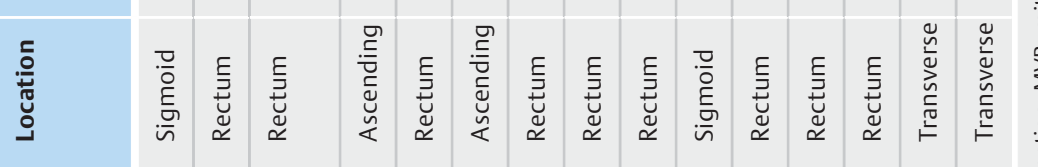

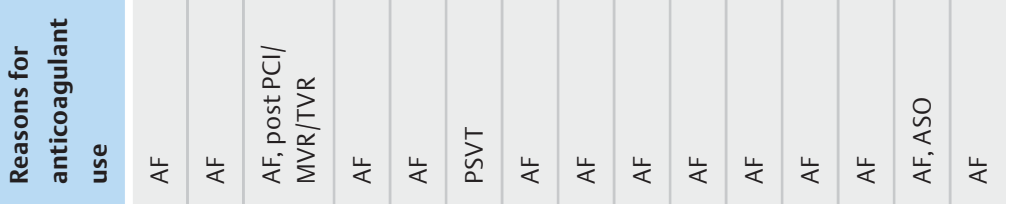

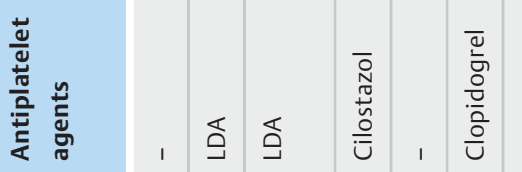

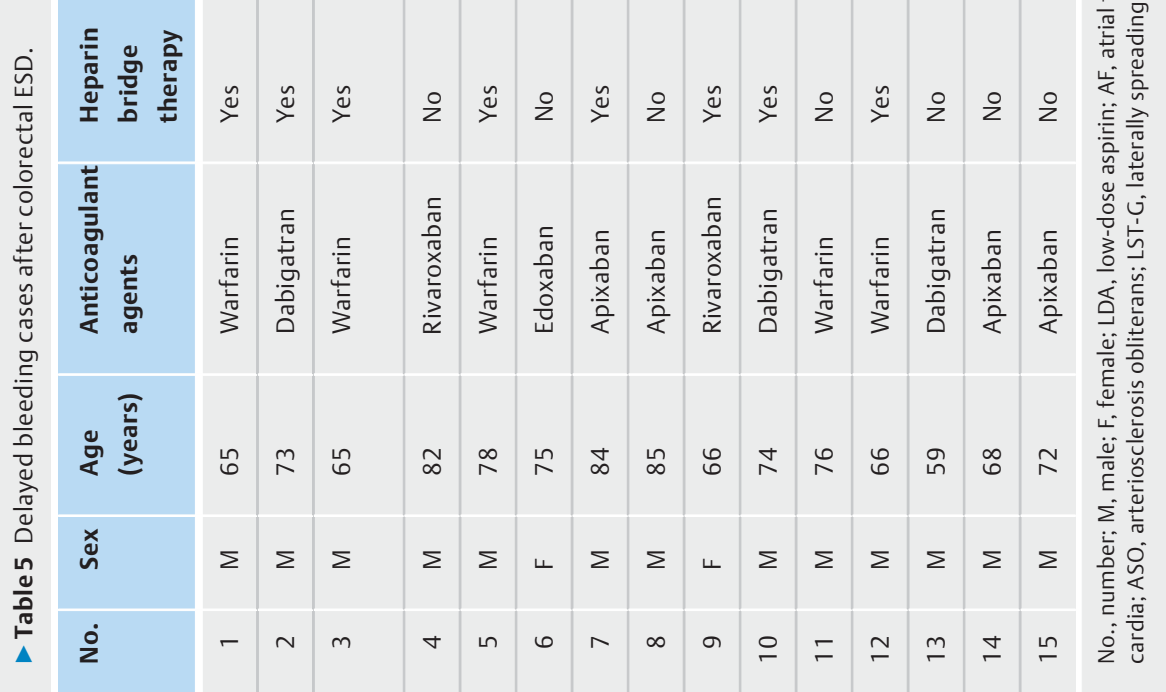




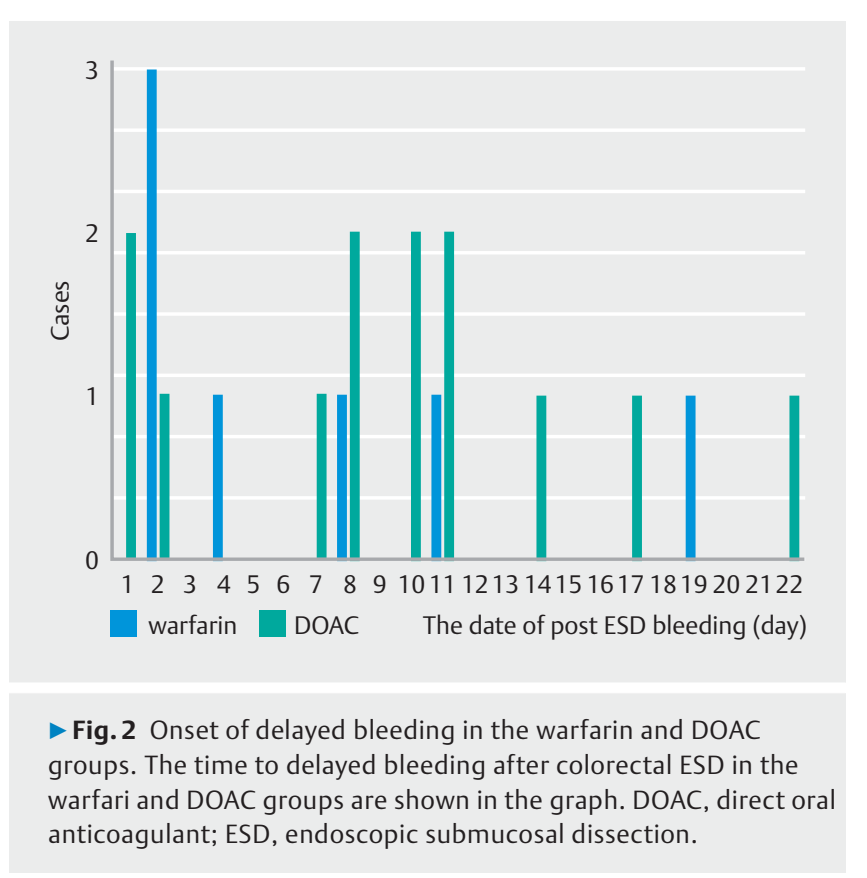

study. Taking into account the high thromboembolic risk [5] without reduction of bleeding risk in the long-term discontinuation of anticoagulant, a short-term withdrawal period for DOAC might be recommended.

In this study, rectal lesions were associated with a higher delayed bleeding rate $(37.1 \%)$ than colonic lesions, similar to the findings in previous reports, which included a small number of patients taking anticoagulants $(1.1 \%-7.3 \%)$ [7-9]. This is the first report to incorporate rectal lesions as a risk factor for delayed bleeding in patients taking anticoagulants.

This study has several limitations. First, it was retrospective study. Second, the timing of anticoagulant interruption and resumption and management of HBT were not unified. Third, there may be a selection bias in management of anticoagulants, because the thrombotic risk varies according to the comorbidities of each patient. Fourth, we could not collect a large patient sample taking anticoagulants even across many institutions. This was due to the small number of patients taking anticoagulants, as previous reports have acknowledged $[14,15]$. Fifth, the number of patients with continued warfarin therapy and those taking edoxaban was small.

\section{Conclusion}

In conclusion, our study revealed that the rate of delayed bleeding was high after colorectal ESD in patients receiving anticoagulant therapy. We therefore need to observe these patients more carefully. Further studies are needed to clarify the difference in delayed bleeding risk among the anticoagulant drugs and the management strategies for the anticoagulants.

\section{Competing interests}

The authors declare that they have no conflict of interest.

\section{References}

[1] Saito Y, Uraoka T, Yamaguchi Y et al. A prospective, multicenter study of 1111 colorectal endoscopic submucosal dissections (with video). Gastrointest Endosc 2010; 72: 1217-1225

[2] Tanaka S, Tamegai Y, Tsuda $S$ et al. Multicenter questionnaire survey on the current situation of colorectal endoscopic submucosal dissection in Japan. Dig Endosc 2010; 22: S2-S8

[3] Takeuchi Y, lishi $\mathrm{H}$, Tanaka $\mathrm{S}$ et al. Factors associated with technical difficulties and adverse events of colorectal endoscopic submucosal dissection: Retrospective exploratory factor analysis of a multicenter prospective cohort. Int J Colorectal Dis 2014; 29: 1275-1284

[4] Odagiri $\mathrm{H}$, Yasunaga $\mathrm{H}$, Matsui $\mathrm{H}$ et al. Hospital volume and the occurrence of bleeding and perforation after colorectal endoscopic submucosal dissection: Analysis of a national administrative database in Japan. Dis Colon Rectum 2015; 58: 597-603

[5] Blacker DJ, Wijdicks EFM, McClelland RL. Stroke risk in anticoagulated patients with atrial fibrillation undergoing endoscopy. Neurology 2003; 61: 964-968

[6] Terasaki M, Tanaka S, Shigita K et al. Risk factors for delayed bleeding after endoscopic submucosal dissection for colorectal neoplasms. Int J Colorectal Dis 2014; 29: 877-882

[7] Ogasawara N, Yoshimine T, Noda $\mathrm{H}$ et al. Clinical risk factors for delayed bleeding after endoscopic submucosal dissection for colorectal tumors in Japanese patients. Eur J Gastroenterol Hepatol 2016; 28: $1407-1414$

[8] Okamoto K, Watanabe T, Komeda Y et al. Risk factors for postoperative bleeding in endoscopic submucosal dissection of colorectal tumors. Oncology 2017; 93: 35-42

[9] Seo M, Song EM, Cho JW et al. A risk-scoring model for the prediction of delayed bleeding after colorectal endoscopic submucosal dissection. Gastrointest Endosc 2019; 89: 990-998.e2

[10] Ninomiya Y, Oka S, Tanaka S et al. Risk of bleeding after endoscopic submucosal dissection for colorectal tumors in patients with continued use of low-dose aspirin. J Gastroenterol 2015; 50: 1041-1046

[11] Hui AJ, Wong RMY, Ching JYL et al. Risk of colonoscopic polypectomy bleeding with anticoagulants and antiplatelet agents: Analysis of 1657 cases. Gastrointest Endosc 2004; 59: 44-48

[12] Beppu K, Osada T, Sakamoto $\mathrm{N}$ et al. Optimal timing for resuming antithrombotic agents and risk factors for delayed bleeding after endoscopic resection of colorectal tumors. Gastroenterol Res Pract 2014; 2014: 825179

[13] Witt DM, Delate T, McCool KH et al. Incidence and predictors of bleeding or thrombosis after polypectomy in patients receiving and not receiving anticoagulation therapy. J Thromb Haemost 2009; 7: 1982-1989

[14] Yoshida N, Naito Y, Murakami T et al. High incidence of postoperative hemorrhage in colorectal endoscopic submucosal dissection during anticoagulant therapy. Int J Colorectal Dis 2016; 31: 1487-1488

[15] Yamashita K, Oka S, Tanaka S et al. Use of anticoagulants increases risk of bleeding after colorectal endoscopic submucosal dissection. Endosc Int Open 2018; 6: E857-E864

[16] Tanaka S, Kashida H, Saito Y et al. JGES guidelines for colorectal endoscopic submucosal dissection/endoscopic mucosal resection. Dig Endosc 2015; 27: 417-434

[17] Fujimoto K, Fujishiro M, Kato M et al. Guidelines for gastroenterological endoscopy in patients undergoing antithrombotic treatment. Dig Endosc 2014; 26: 1-14

[18] Kato M, Uedo N, Hokimoto $S$ et al. Guidelines for gastroenterological endoscopy in patients undergoing antithrombotic treatment: 2017 appendix on anticoagulants including direct oral anticoagulants. Dig Endosc 2018; 30: 433-440 
[19] Acosta RD, Abraham NS, Chandrasekhara V et al. The management of antithrombotic agents for patients undergoing Gl endoscopy. Gastrointest Endosc 2016; 83: 3-16

[20] Veitch AM, Vanbiervliet G, Gershlick AH et al. Endoscopy in patients on antiplatelet or anticoagulant therapy, including direct oral anticoagulants: British Society of Gastroenterology (BSG) and European Society of Gastrointestinal Endoscopy (ESGE) guidelines. Gut 2016; 65: $374-389$

[21] Matsuo S, Imai E, Horio M et al. Revised equations for estimated GFR from serum creatinine in Japan. Am J Kidney Dis 2009; 53: 982-992

[22] Harada H, Nakahara R, Murakami D et al. The effect of anticoagulants on delayed bleeding after colorectal endoscopic submucosal dissection. Surg Endosc 2020; 34: 3330-3337

[23] Desai J, Granger CB, Weitz JI et al. Novel oral anticoagulants in gastroenterology practice. Gastrointest Endosc 2013; 78: 227-239

[24] Nagata N, Yasunaga H, Matsui $\mathrm{H}$ et al. Therapeutic endoscopy-related GI bleeding and thromboembolic events in patients using warfarin or direct oral anticoagulants: Results from a large nationwide database analysis. Gut 2018; 67: 1805-1812

[25] Holster IL, Valkhoff VE, Kuipers EJ et al. New oral anticoagulants increase risk for gastrointestinal bleeding: A systematic review and meta-analysis. Gastroenterology 2013; 145: 105-112.e15

[26] Yoshio T, Nishida T, Kawai N et al. Gastric ESD under heparin replacement at high-risk patients of thromboembolism is technically feasible but has a high risk of delayed bleeding: Osaka University ESD Study Group. Gastroenterol Res Pract 2013; 2013: 365830

[27] Inoue T, Nishida T, Maekawa A et al. Clinical features of post-polypectomy bleeding associated with heparin bridge therapy. Dig Endosc 2014; 26: 243-249
[28] Jaruvongvanich V, Assavapongpaiboon B, Wijarnpreecha K et al. Heparin-bridging therapy and risk of post-polypectomy bleeding: Metaanalysis of data reported by Japanese colonoscopists. Dig Endosc 2017; 29: 743-748

[29] Lin D, Soetikno RM, McQuaid K et al. Risk factors for postpolypectomy bleeding in patients receiving anticoagulation or antiplatelet medications. Gastrointest Endosc 2018; 87: 1106-1113

[30] Sanomura Y, Oka S, Tanaka S et al. Taking warfarin with heparin replacement and direct oral anticoagulant is a risk factor for bleeding after endoscopic submucosal dissection for early gastric cancer. Digestion 2018; 97: 240-249

[31] Beyer-Westendorf J, Gelbricht V, Forster K et al. Peri-interventional management of novel oral anticoagulants in daily care: results from the prospective Dresden NOAC registry. Eur Heart J 2014; 35: 1888 1896

[32] Douketis JD, Healey JS, Brueckmann M et al. Perioperative bridging anticoagulation during dabigatran or warfarin interruption among patients who had an elective surgery or procedure. Substudy of the RE-LY trial. Thromb Haemost 2015; 113: 625-632

[33] Radaelli F, Fuccio L, Paggi S et al. Periendoscopic management of direct oral anticoagulants: A prospective cohort study. Gut 2019; 68: 969-976

[34] Douketis JD, Spyropoulos AC, Kaatz S et al. Perioperative bridging anticoagulation in patients with atrial fibrillation. N Engl J Med 2015; 373: $823-833$

[35] Schulman S, Carrier M, Lee AYY et al. Perioperative management of dabigatran: A prospective cohort study. Circulation 2015; 132: 167173 
A total of 107 patients performed colorectal ESD from April 2010 to December 2017

Excluded:

- performed prophylactic clip closure

(18 patients)

- needed operation due to delayed perforation (2 patients)

87 patients analyzed

- Supplementary Fig. 1 Flowchart of patients included in this study. ESD, endoscopic submucosal dissection. 